\title{
The recruitment of
} different compartments within a muscle depends on the mechanics of the movement

\author{
James M. Wakeling* \\ School of Kinesiology, Simon Fraser University, Burnaby, \\ British Columbia, Canada V5A 1 S6 \\ *wakeling@sfu.ca
}

Muscles are commonly assumed to have uniform activations across their bellies. Yet animal studies have shown that different regions across a muscle can vary in their architecture, fibre type, levels of activation and the transfer of forces to the bones and tendons. The purpose of this study was to test whether regional variations in activity occurred across the soleus, medial gastrocnemius and lateral gastrocnemius in man, and whether these regional variations changed with altered mechanical demands on the limb. Arrays of surface electrodes were placed over these muscles and the electromyograms (EMG) measured for a range of cycling tasks where the resistance and pedal velocity was independently altered. Significant variations in the magnitude and timing of the EMG occurred across these muscles, which were most pronounced in the gastrocnemii and occurred in both the proximodistal and mediolateral directions. The patterns of variation across the muscles changed in response to the altered mechanical demands during the cycling. It is likely that the muscle fascicles in the gastrocnemii contribute varied mechanical functions to the contractions that depend on both their location within the muscle belly and on the mechanical requirements of the movement.

Keywords: muscle function; electromyography; biomechanics

\section{INTRODUCTION}

The force developed by a muscle fibre depends in part on its level of activity, orientation, fibre type and strain and these factors can vary between regions within a muscle. For instance, regional variations occur in muscle activation (English 1984; Hoffer et al. 1987; Pratt \& Loeb 1991; Schieber 1993; Soman et al. 2005; Wakeling et al. 2007), muscle fascicle orientation (Ritruechai et al. 2008), fibre type (English \& Letbetter 1982; Wang \& Kernell 2000) and strain (Chanaud et al. 1991; Pappas et al. 2002; Soman et al. 2005; Higham et al. 2008). Regional variations in activity and force production result in changes to the net force vectors produced by the whole muscle (Schieber 1993; Carrasco et al. 1999). It is therefore likely that different regions of a muscle

can have different functions, even within the same movement. Furthermore, it has been inferred that the fractional work performed by different regions of a muscle varies in response to movement behaviours for both the medial gastrocnemius (MG) during walking (Higham et al. 2008) and the pectoralis during flying (Soman et al. 2005).

The assessment of muscle function in humans is often aided by surface electromyography (sEMG) to quantify muscle activity patterns. The spectral properties of the sEMG signal vary with the proximity of the electrodes to the muscle boundaries and the innervation zone (DeLuca 1997) and so standardized electrode placements are commonly used for each muscle (Hermens et al. 2000). Such an approach makes the implicit assumption that measurements from one location are representative of the whole muscle belly. However, this assumption may not hold true, particularly across a range of mechanical demands because the optimal activation may involve specific regions of the muscle as has been observed in animal studies (reported above).

The purpose of this study was to quantify the differences in magnitude and timing of the sEMG in different regions of the ankle extensor muscles in man. These regional variations were identified across a range of mechanical demands on the leg in order to maximize regional changes in muscle function.

\section{MATERIAL AND METHODS}

\section{(a) Subjects and protocol}

Six male cyclists (mass $82.2 \pm 5.5 \mathrm{~kg}$ and age $36.8 \pm 3.0$ years) were tested on a stationary bicycle (indoor trainer: Schoberer Rad Meßtechnik, Jülich, Germany, equipped with torque sensing cranks: SRM Powermeter). Each cyclist completed a 5 min warmup period and was then tested for nine conditions presented thrice in a randomized block format $(60,80,100,120$ or $140 \mathrm{~g}$ (revolutions $\min ^{-1}$ ) at a crank torque of $6.5 \mathrm{~N} \mathrm{~m}$ and additionally crank torques of $12.9,25.1,32.4$ and $39.9 \mathrm{Nm}$ at a pedal cadence of $60 \mathrm{~g}$ ). Data were recorded for $30 \mathrm{~s}$ of steady cycling for each condition with a rest of $45 \mathrm{~s}$ between conditions.

\section{(b) Measurement of muscle activity}

sEMG was recorded from the lateral gastrocnemius (LG), MG and the soleus, Sol, from the left leg at $2000 \mathrm{~Hz}$ using bipolar $\mathrm{Ag} / \mathrm{AgCl}$ sEMG electrodes and Biovision amplifiers (Wehrheim, Germany). For each of the gastrocnemii, the muscle belly was divided into quadrants (when the ankle was extended) that separated proximal/ distal and medial/lateral regions; an electrode recorded electromyograms (EMG) from each quadrant. Two electrodes were used to record EMG from the medial and lateral sides of the Sol. A pedal switch provided timing information for each pedal revolution. Data were recorded for at least 30 revolutions in each trial.

\section{(c) Data analysis}

The EMG intensities were calculated using wavelet analysis (von Tscharner 2000) where the intensity is a close approximation of the power of the signal. Cross-correlations were calculated for each trial between every pairing of electrodes for the raw (unrectified) EMG and for the EMG intensities. EMG intensities were normalized to the mean EMG intensity recorded across all trials for each muscle, and interpolated to 100 points per pedal revolution. Principal component (PC) analysis determined the common features and differences of the EMG intensity traces (for each pedal revolution) and were calculated separately for each muscle. Data were analysed using MathEMATICA software v. 6 (Wolfram Research Inc., Champaign, IL, USA). The effects of pedal cadence and crank torque (covariates), subject (random factor) and muscle region (factor) on the PC I and PC II loading scores were determined using general linear model MANCOVA using MiNITAB software v. 13 (Minitab Inc., State College, PA, USA). 
Table 1. Correlations in the EMG between different recording sites. (Correlations, $r^{2}$, were calculated from a cross-correlation of either the raw EMG signals (lower left values) or the EMG intensities (italics, upper right values). The $r^{2}$ values are the mean from all trials and all subjects. Subscripts denote different muscle regions: p, proximal; d, distal; m, medial; 1, lateral.)

\begin{tabular}{lllllllllll}
\hline & $\mathrm{LG}_{\mathrm{p}, \mathrm{l}}$ & $\mathrm{LG}_{\mathrm{p}, \mathrm{m}}$ & $\mathrm{LG}_{\mathrm{d}, \mathrm{l}}$ & $\mathrm{LG}_{\mathrm{d}, \mathrm{m}}$ & $\mathrm{MG}_{\mathrm{p}, 1}$ & $\mathrm{MG}_{\mathrm{p}, \mathrm{m}}$ & $\mathrm{MG}_{\mathrm{d}, \mathrm{p}}$ & $\mathrm{MG}_{\mathrm{d}, \mathrm{m}}$ & $\mathrm{Sol}_{1}$ & $\mathrm{Sol}_{\mathrm{m}}$ \\
\hline $\mathrm{LG}_{\mathrm{p}, 1}$ & - & 0.45 & 0.46 & 0.44 & 0.29 & 0.30 & 0.30 & 0.30 & 0.25 & 0.27 \\
$\mathrm{LG}_{\mathrm{p}, \mathrm{m}}$ & 0.01 & - & 0.48 & 0.50 & 0.32 & 0.32 & 0.32 & 0.34 & 0.27 & 0.28 \\
$\mathrm{LG}_{\mathrm{d}, \mathrm{l}}$ & 0.02 & 0.00 & - & 0.52 & 0.30 & 0.30 & 0.30 & 0.30 & 0.26 & 0.28 \\
$\mathrm{LG}_{\mathrm{d}, \mathrm{m}}$ & 0.01 & 0.05 & 0.09 & - & 0.32 & 0.32 & 0.34 & 0.34 & 0.28 & 0.29 \\
$\mathrm{MG}_{\mathrm{p}, \mathrm{l}}$ & 0.02 & 0.04 & 0.01 & 0.04 & - & 0.56 & 0.58 & 0.58 & 0.41 & 0.42 \\
$\mathrm{MG}_{\mathrm{p}, \mathrm{m}}$ & 0.00 & 0.00 & 0.00 & 0.01 & 0.01 & - & 0.58 & 0.64 & 0.44 & 0.45 \\
$\mathrm{MG}_{\mathrm{d}, 1}$ & 0.01 & 0.00 & 0.00 & 0.00 & 0.01 & 0.01 & - & 0.62 & 0.42 & 0.44 \\
$\mathrm{MG}_{\mathrm{d}, \mathrm{m}}$ & 0.00 & 0.01 & 0.01 & 0.00 & 0.04 & 0.05 & 0.00 & - & 0.44 & 0.46 \\
$\mathrm{Sol}_{1}$ & 0.01 & 0.00 & 0.01 & 0.01 & 0.00 & 0.01 & 0.00 & 0.01 & - & 0.61 \\
$\mathrm{Sol}_{\mathrm{m}}$ & 0.01 & 0.00 & 0.00 & 0.00 & 0.01 & 0.02 & 0.01 & 0.01 & 0.01 & - \\
\hline
\end{tabular}
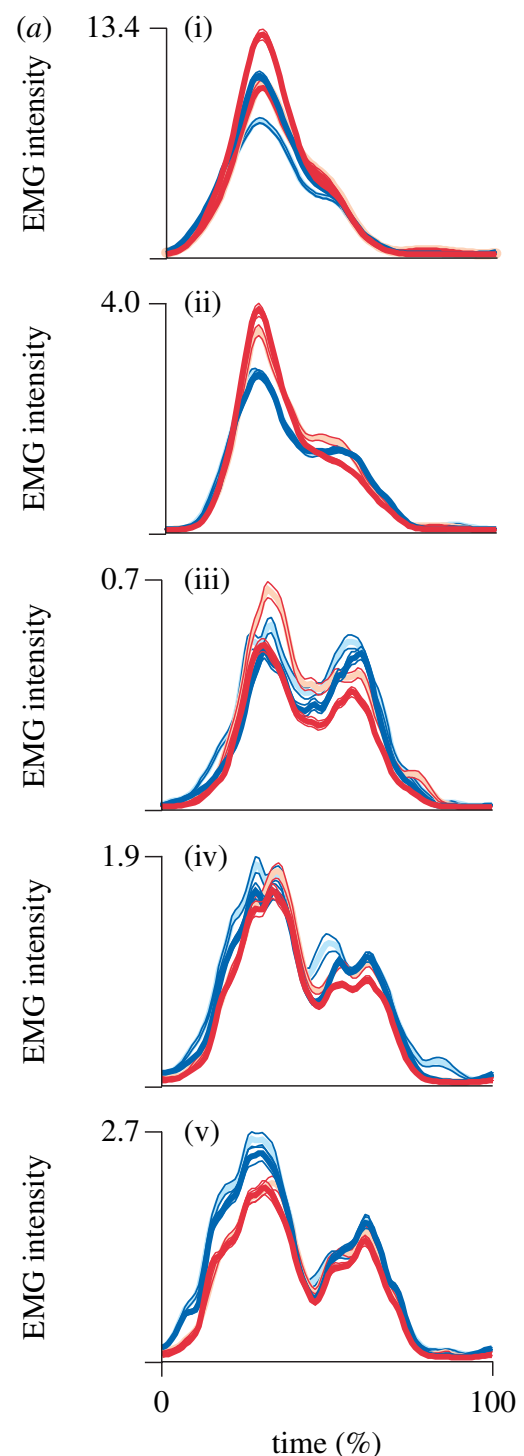

(b) 12.7

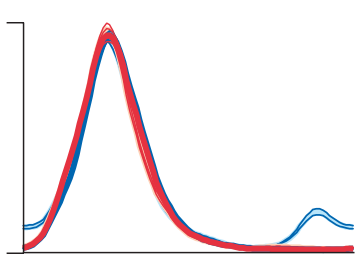

5.7

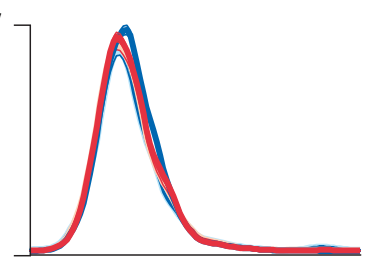

2.0

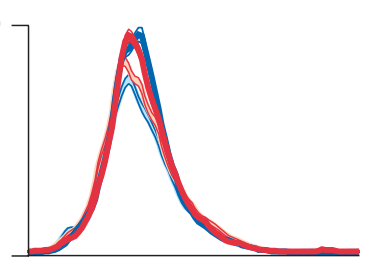

2.7

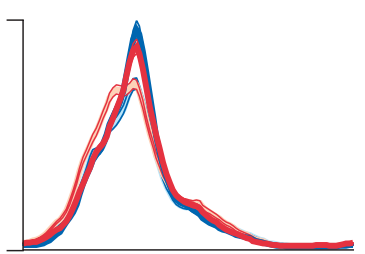

2.5

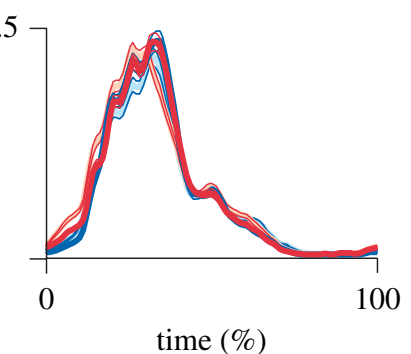

(c) 8.9

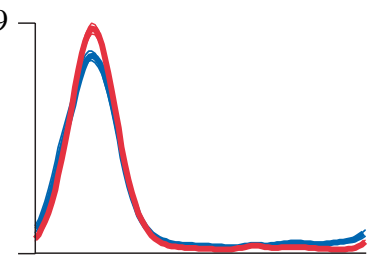

2.7

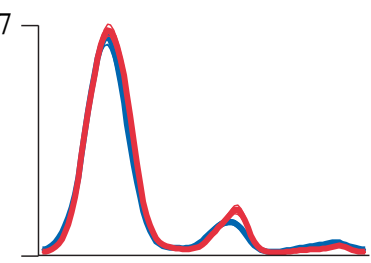

0.9
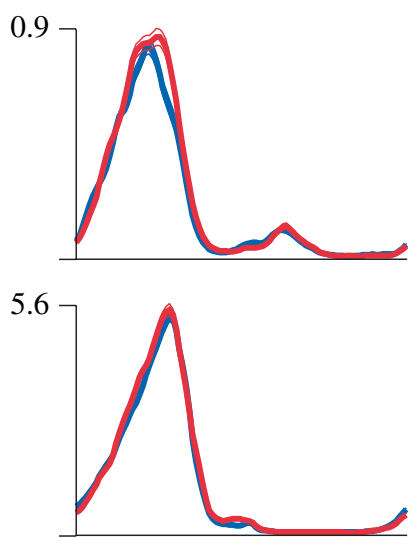

8.3

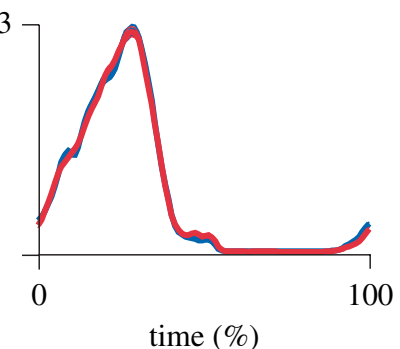

Figure 1. Regional changes in EMG intensity for the (a) LG, (b) MG and (c) Sol when cycling at increasing crank torques. Data show the mean (thick lines) \pm s.e.m. (thin lines) averaged over 504 pedal revolutions. Lateral and medial recording sites are denoted by blue and red colours, respectively. Proximal and distal sites are denoted by light and dark colours, respectively. (i) $140 g, 6.5 \mathrm{~N} \mathrm{~m}$; (ii) $100 g, 6.5 \mathrm{~N} \mathrm{~m}$; (iii) $60 g, 6.5 \mathrm{~N} \mathrm{~m}$; (iv) $60 g, 25.1 \mathrm{~N} \mathrm{~m}$; (v) $60 g, 39.9 \mathrm{~N} \mathrm{~m}$.

\section{RESULTS AND DISCUSSION}

There was very little correlation between the raw EMG signals from different compartments within the same muscle (table 1). For both the LG and MG, the maximum $r^{2}$ value from the cross-correlations between adjacent compartments was 0.09 , indicating that there was very little common signal recorded in the different regions and thus that different populations of motor units were recorded in the different regions. There was even less correlation 


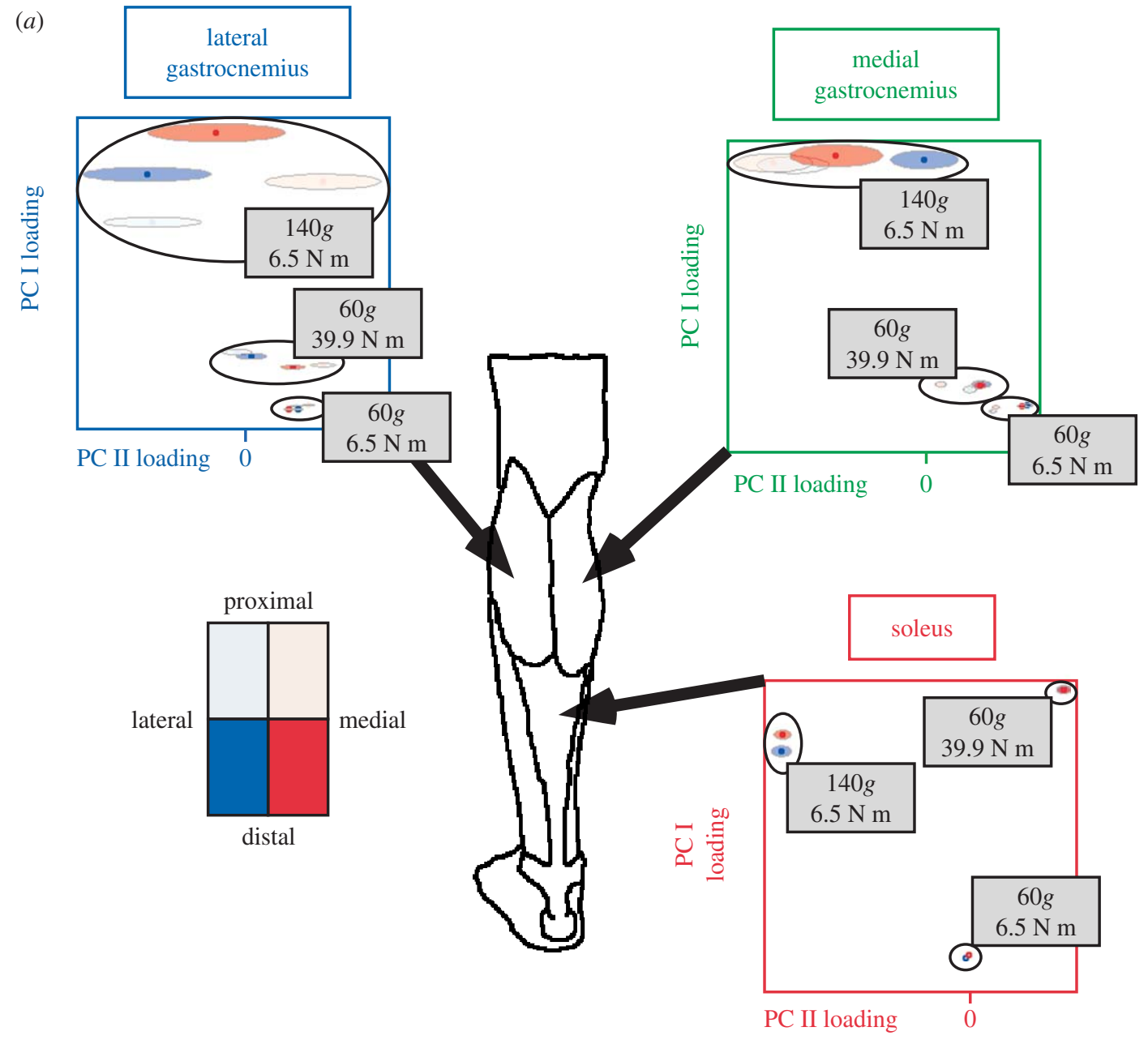

(b)

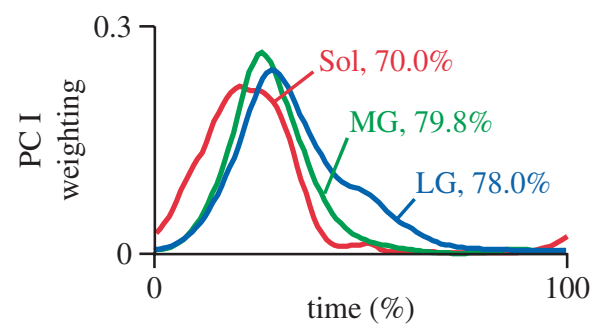

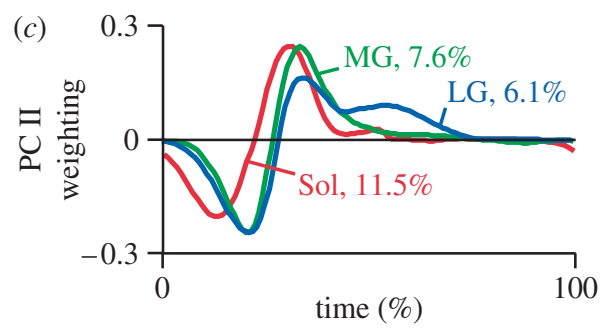

Figure 2. Differences in the patterns of activity from the muscle regions for the varied mechanical demands. (a) PC loading score plots for the patterns of activity are shown for each muscle. Each point shows the mean $(n=504)$ loading score, and the coloured ellipses show the $95 \%$ confidence limits for these means. Lateral and medial recording sites are denoted by blue and red colours, respectively. Proximal and distal sites are denoted by light and dark colours, respectively. Data are grouped together and shown for the three extreme mechanical regimes that were performed. (b) PC I loading score is on the vertical and represents levels of activity, with greater scores being associated with greater muscle activity. (c) PC II loading scores are on the horizontal and represent a shift in timing, with more positive scores representing later timing of muscle activity. The percentage of the signals explained by each PC is shown $(b, c)$.

between adjacent muscles, with the greatest $r^{2}$ being 0.04 from the cross-correlations between the medial-proximal region of LG and the lateralproximal region of the MG. In general, the signals between and within the muscles could thus be considered free from crosstalk from neighbouring regions.

The MG, LG and Sol have a common function of ankle extension and so it should be expected that their activity patterns show common elements. The EMG intensity provides a time-filtered envelope that is related to the muscle activity: the time resolution of the intensity is of the order of $20 \mathrm{~ms}$ (von Tscharner 2000) and thus is sensitive to fluctuations in the motor unit activity. The cross-correlations of the EMG intensities from different regions between muscles (table 1) had an average $r^{2}$ of 0.33 , and thus the majority of the activity patterns in the MG, LG and Sol were independent from each other. The greatest cross-correlation between EMG intensities from adjacent regions within the same muscle (table 1) had an $r^{2}$ of 0.64 , and the average $r^{2}$ between adjacent regions within a muscle was 0.54 . The different populations of motor units recorded from the different regions within each muscle thus had little more than half of their activity patterns in common. Thus a substantial portion of the motor 
unit activities were independent between the different muscle regions.

All three muscles showed a burst of EMG intensity that peaked within the first half of the pedal cycle (figure 1). At the highest cadence trials, the LG showed graded levels of EMG intensity from the distal-medial region decreasing to the proximal-lateral region and the Sol similarly had EMG intensity decreasing from the medial to lateral sides. At the slow cadence-low torque trials, the LG showed a proximal to distal gradient for its first peak of EMG intensity, but lateral to medial gradient for the second burst; the MG showed a single burst of EMG intensity for these trials that had a distal to proximal gradient of EMG intensity, and the Sol was most similar between its different regions. For the highest torque trials, the LG showed a lateral to medial gradient of EMG intensity, whereas the MG and Sol were more similar in EMG intensities between their regions. Changes in the relative levels of activity between different regions of the muscles are most apparent in the gastrocnemii and these regional patterns of activity are modulated by the mechanical demands on the leg. Regional differences in muscle architecture have been demonstrated in the quadriceps muscles in man (Blazevich et al. 2006), although no such data are available for the ankle extensors. However, it is possible that the regional variations in muscle activity observed in this study would result in regional differences in the function of the muscle fascicles.

The first two PCs capture the main information from the EMG intensities (figure 2). The first PC weighting explained the general pattern of activity and its loading score correlated with the EMG intensity with an $r^{2}$ between 0.94 and 0.96 for the three muscles. The second PC weighting explained a time-shift in the EMG intensity: a relatively greater PC II to I loading score ratio corresponded to a later timing of the burst of EMG intensity. The PC loading score plots (figure 2) illustrate how the EMG activity profiles differ between the muscle regions for the different mechanical conditions. The LG showed the most pronounced differences in both activity (PC I loading score) and timing (ratio of PC II to I loading scores). The region with the greatest activity changed between the highest velocity trial (medial-distal LG) and the highest torque trial (proximal-lateral LG), indicating that these regional gradations of activity must be under neuromechanical control.

The data from this study show that activity patterns across the ankle extensor muscles in man follow similar regionalization to those previously reported for animals. Muscle fascicle strains can be non-uniform across a muscle (Pappas et al. 2002) due to curvature within the fascicles (Blemker et al. 2005). It is possible that local fascicle strains and activities are linked via the local action of stretch reflexes from the muscle spindles, but we do not know the mechanisms that control regional variations in activity. It is likely that the fascicles from different regions of these muscles generate different forces and strains and thus contribute different functions to the whole muscle when it is faced with a range of mechanical tasks. However, modelling studies that simulate the forces generated during muscle contractions typically treat the muscle as a homogeneous unit (Zajac 1989). It will be important to determine the extent to which regional variations in architecture, biochemistry and activation affect the function of a whole muscle.

The experimental procedures were approved by the Office for Research Ethics at Simon Fraser University.

Financial support was provided by NSERC. The author expresses thanks to Tamara Horn for invaluable help with the data collection.

Blazevich, A. J., Gill, N. D. \& Zhou, S. 2006 Intra- and intermuscular variation in human quadriceps femoris architecture assessed in vivo. F. Anat. 209, 289-310. (doi:10.1111/j.1469-7580.2006.00619.x)

Blemker, S. S., Pinsky, P. M. \& Delp, S. L. 2005 A 3D model of muscle reveals the causes of non-uniform strains in the biceps brachii. F. Biomech. 38, 657-665. (doi:10.1016/j.jbiomech.2004.04.009)

Carrasco, D. J., Lawrence III, J. \& English, A. W. 1999 Neuromuscular compartments of cat lateral gastrocnemius produce different torques about the ankle joint. Motor Control 3, 436-446.

Chanaud, C. M., Pratt, C. A. \& Loeb, G. E. 1991 Functionally complex muscles of the cat hindlimb II. Mechanical and architectural heterogeneity within the biceps femoris. Exp. Brain Res. 85, 257-270. (doi:10. 1016/S0079-6123(08)62684-9)

DeLuca, C. J. 1997 The use of surface electromyography in biomechanics. F. Appl. Biomech. 13, 135-136.

English, A. W. 1984 An electromyographic analysis of compartments in cat lateral gastrocnemius muscle during unrestrained locomotion. F. Neurophysiol. 52, 114-125. (doi:10.1002/ar.1092040205)

English, A. W. \& Letbetter, W. D. 1982 A histochemical analysis of identified compartments in cat lateral gastrocnemius muscle. Anat. Rec. 204, 123-130. (doi:10.1002/ ar.1092040205)

Hermens, H. J., Freriks, B., Disselhorst-Klug, C. \& Rau, G. 2000 Development of recommendations for sEMG sensors and sensor placement procedures. F. Electromyogr. Kinesiol. 10, 361-374. (doi:10.1016/S1050-6411 (00)00027-4)

Higham, T. E., Biewener, A. A. \& Wakeling, J. M. 2008 Functional diversification within and between muscle synergists during locomotion. Biol. Lett. 4, 41-44. (doi: 10.1098/rsbl.2007.0472)

Hoffer, J. A., Loeb, G. E., Sugano, N., Marks, W. B., O’Donovan, M. J. \& Pratt, C. A. 1987 Cat hindlimb motoneurons during locomotion. III. Functional segregation in sartorius. F. Neurophysiol. 57, 554-562.

Pappas, G. P., Asakawa, D., Delp, S. L., Zajac, F. E. \& Drace, J. E. 2002 Nonuniform shortening in the biceps brachii during elbow flexion. F. Appl. Physiol. 92, 2381-2389.

Pratt, C. A. \& Loeb, G. E. 1991 Functionally complex muscles of the cat hindlimb I. Patterns of activation across sartorius. Exp. Brain Res. 85, 243-256.

Ritruechai, P., Weller, R. \& Wakeling, J. M. 2008 Regional variations in muscle anatomy in the equine longissimus dorsi. Equine Vet. F. 40, 246-251. 
Schieber, M. H. 1993 Electromyographic evidence of two functional subdivisions in the rhesus monkey's flexor digitorum profundus. Exp. Brain Res. 95, 251-260. (doi:10.1007/BF00229783)

Soman, A., Hedrick, T. L. \& Biewener, A. A. 2005 Regional patterns of pectoralis fascicle strain in the pigeon Columbia livia during level flight. F. Exp. Biol. 208, 771-786. (doi:10.1242/jeb.01432)

von Tscharner, V. 2000 Intensity analysis in time-frequency space of surface myoelectric signals by wavelets of specified resolution. F. Electromyogr. Kinesiol. 10, 433-445. (doi:10.1016/S1050-6411(00)00030-4)
Wakeling, J. M., Ritruechai, P., Dalton, S. \& Nankervis, K. 2007 Segmental variation in the activity and function of the equine longissimus dorsi muscle during walk and trot. Equine Comp. Exercise Physiol. 4, 95-103. (doi:10. 1017/S1478061507812126)

Wang, L. C. \& Kernell, D. 2000 Proximo-distal organisation and fibre type regionalization in rat hindlimb muscles. F. Mus. Res. Cell Motil. 21, 587-598. (doi:10. 1023/A:1026584307999)

Zajac, F. E. 1989 Muscle and tendon: properties, models, scaling, and application to biomechanics and motor control. Crit. Rev. Biomed. Eng. 17, 359-411. 